\title{
Comparative study on Content based Image Retrieval based on Gabor Texture Features at Different Scales of Frequency and Orientations
}

\author{
S. Mangijao Singh \\ Dept. of computer Science, Assam \\ Raju Rajkumar \\ Dept. of computer science, \\ University, Silchar India, Pin code - Assam University, Silchar India, Pin \\ 788011 \\ code - 788011 \\ K. Hemachandran \\ Dept. of computer science, \\ Assam University, Silchar India, Pin \\ code - 788011
}

\begin{abstract}
Content-Based Image Retrieval (CBIR) systems help users to retrieve relevant images based on their contents such as color and texture. In this paper, a study has been made on the application of Gabor Wavelet Transform for texture classification at different values of the number of scales(S) and the number of orientations (K). Texture features are found by calculating the mean and variation of Gabor filtered image. The image indexing and retrieval are conducted on natural images. Based on experiments, Gabor wavelet at five scales of frequency and four orientations gives better performance than the other commonly used scales and orientations i.e., three scales and four orientations, three scales and six orientations, four scales and five orientations, four scales and six orientations and five scales and six orientations.
\end{abstract}

\section{Keywords}

CBIR, Gabor wavelet, Canberra distance, Texture.

\section{INTRODUCTION}

Like color, image texture is also very important. Texture is an important feature of natural images and texture provides important characteristics for surface and object identification from image [1]. Texture analysis is a major component of image processing and it is fundamental to many applications such as remote sensing, quality inspection, medical imaging, etc.

A variety of techniques have been developed for measuring texture similarity. In most of the techniques, the values of what are known as second-order statistics are calculated from query and stored images [2] and compared. In these methods, measures of image texture such as the degree of contrast, coarseness, directionality and regularity $[3,4]$, directionality and randomness[5] are calculated. Some other methods of texture analysis for image retrieval have used Gabor filters [6] and fractals [7].

Gabor filter has been used widely to extract texture features from the images for image retrieval [ 6,8, 9, 10, 11, 12], and has been shown to be very efficient. Manjunath and Ma [6] have shown that image retrieval using Gabor features outperforms that using Pyramid-structured wavelet transform
(PWT) features, tree-structured wavelet transform(TWT) features and multiresolution simultaneous autoregressive model (MR-SAR) features.

Basically Gabor filters are a group of wavelets, with each wavelet capturing energy at a specific frequency and specific orientation. Expanding a signal using this basis provides a localized frequency description and captures local feature/energy of the signal. From this group of energy distributions, texture features can be constructed. The scale (frequency) and orientation tunable property of Gabor filter makes it especially useful for texture analysis. Experimental evidence on human and mammalian vision supports the notion of spatial-frequency (multi-scale) analysis that maximizes the simultaneous localization of energy in both spatial and frequency domains[13]

With the advent of multimedia techniques, the image data has grown exponentially and this has compelled the need for innovative tools which can easily manage, retrieve and visualize images from the large multimedia databases. Calvin Moores proposed Information Retrieval [14] in 1951. In this process, information was organized and stored according to a certain way, and in accordance with the needs of users to find the interrelated information. It is also called "Information Storage and Retrieval". However, at that time, the retrieval was based on the text of the document. By age 70, database experts began to study how to manage data effectively. The main philosophy in text based image retrieval is to establish a relation between a Keyword or text description of the title as well as some additional information and the storage path of the image. However, with the growing storage capacity of databases, the text-based image retrieval technology lead to certain difficulties like: 1 . it has become impossible to note each and every image in the database; 2 . the subjectivity and non-precision of image annotation may lead to ambiguity in the retrieval process. In order to overcome these problems, Content based image retrieval (CBIR) was proposed in the 90's.

Because of the proliferation of video and image data in digital form, Content-based image retrieval (CBIR) [15] has become a prominent research topic. The increased bandwidth 
availability to access the internet in the near future will allow the users to search for and browse through video and image databases located at remote sites. Therefore, fast retrieval of images from large databases is an important problem that needs to be addressed. The desired characteristics of CBIR systems are High retrieval efficiency and less computational complexity. In content-based image retrieval (CBIR) system, images would be indexed by their visual content such as color, texture, shape etc. and the desired images are retrieved from a large collection, on the basis of features that can be automatically extracted from the images themselves [16]. Considerable research work has been done to extract these low level image features [16 and 17], evaluate distance metrics, and look for efficient searching schemes [18 and 19]. Basically, most CBIR systems work in the same way: A feature vector is extracted from each image in the database and the set of all feature vectors is organized as a database index. At query time, a feature vector is extracted from the query image and it is matched against the feature vectors in the index. The crucial difference between the various systems lies in the features that they extract and in the algorithms that are used to compare feature vectors. The block diagram of a basic CBIR system is shown in Fig. 1.

In the whole retrieval process, feature extraction is crucial [20], it is closely related to all aspects of the future. In recent years, many scholars began to study high-level semantic features of images. In practice, a feature or a combination of several features is often used to search for images [21] and in this study the feature vectors based on Gabor texture are used to search for images.

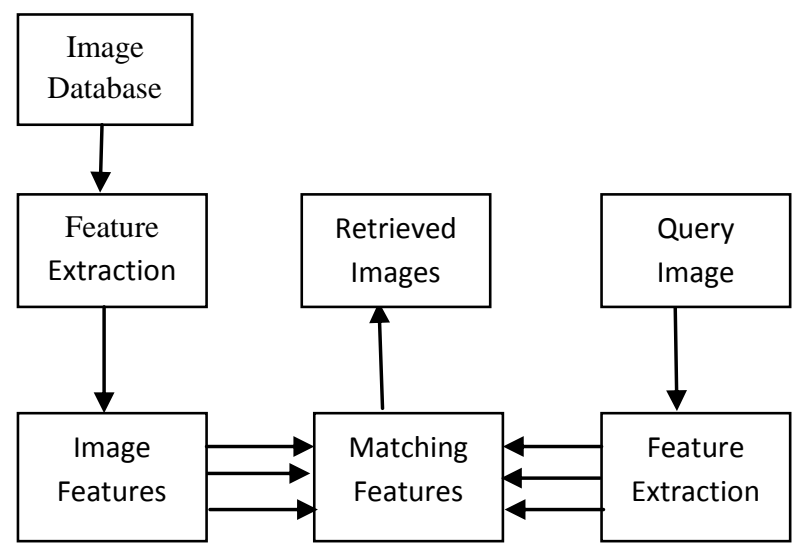

Fig. 1: Block diagram of CBIR

Color, texture, local shape and spatial information in a variety of forms are the most widely used features in such systems [22]-[25]. Because of high demand for searching image databases of ever-growing size, CBIR is becoming very popular. As speed and precision are important, it is needed to develop a system for retrieving images that is efficient.

Choras et al. [26] proposed an integrated color, texture and shape feature extraction method in which Gabor filtration is used for determining the number of regions of interest (ROIs). They calculated texture and color features from the ROIs based on threshold Gabor features and histograms, color moments in YUV space, and shape features based on Zernike moments. The features presented proved to be efficient in determining similarity between images.
Huang et al have proposed a method based on color and texture features [27]. They have used color moments of the Hue, Saturation and Value (HSV) of the image as color feature, and Gabor descriptors as texture features. They assigned weights to each feature and calculated the similarity with combined features of color and texture using normalized Euclidean distance. They reported that the proposed method has higher retrieval accuracy than the conventional method using color and texture features. The feature dimension is also lower than the conventional methods.

Maheshwari et al. [28] have used Color moment and Gabor filter to extract features for image dataset. In their study, Kmeans and hierarchical clustering algorithm is applied to group the image dataset into various clusters.

A content based image retrieval (CBIR) which combines color and texture features is proposed [29]. They reported that the retrieval results obtained by applying HSV color Histogram + Gabor Wavelet Transform demonstrated significant improvement in precision and recall compared to RGB color Histogram + Standard Wavelet Transform.

A comparison on CBIR based by Color Histogram, Gabor and Wavelet Transform is carried out in [30].. The experiments demonstrate that more images are retrieved by using Gabor than the other two feature extraction techniques, Color Histogram, Wavelet Transform.

Many authors have used different values of the number of scales(S) and the number of orientations (K) $[29,30,33,34,35]$ to extract texture features using Gabor filter. In their study, no mention is made about why they have chosen a specific scale and orientation. In this paper, an attempt has been made to study more combinations of scales and orientations.

The remainder of the paper is organized as follows: in section 2, fundamentals of 2-D Gabor filter is described. Section 3 describes texture feature extraction and similarity measurements. Section 4 describes the experiments and results. Finally, the conclusions are presented in section 5 .

\section{Gabor filter}

Texture is defined as structure of surfaces formed by repeating a particular element or several elements in different relative spatial positions [31]. Generally, the repetition involves local variations of scale, orientation, or other geometric and optical features of the elements. Image textures are defined as images of natural textured surfaces and artificially created visual patterns. It contains important information about the structural arrangement of the surface i.e., clouds, leaves, bricks, fabric, etc. It also describes the relationship of the surface to the surrounding environment. It is a feature that describes the distinctive physical composition of a surface.

Gabor wavelet is widely adopted to extract texture from the images and has been shown to be very efficient. Basically Gabor filters are a group of wavelets, with each wavelet capturing energy at a specific frequency and specific orientation. The scale and orientation tunable property of Gabor filter makes it especially useful for texture analysis. The design of the Gabor filter for texture analysis is explained by different authors [32 and 33]

For a given image $\mathrm{I}(\mathrm{x}, \mathrm{y})$ with size (PXQ), the discrete Gabor wavelet transform is given by a convolution: 
$\mathrm{G}_{\mathrm{mn}}(\mathrm{x}, \mathrm{y})=\sum_{\mathrm{s}} \sum_{\mathrm{t}} \mathrm{I}(\mathrm{x}-\mathrm{s}, \mathrm{y}-\mathrm{t}) \psi_{\mathrm{mn}}^{*}(\mathrm{~s}, \mathrm{t})$

Where, $\mathrm{s}$ and $\mathrm{t}$ are the filter mask size variables, and $\psi_{\mathrm{mn}}^{*}$ is the complex conjugate of $\psi_{\mathrm{mn}}$ which is a class of self-similar functions generated from dilation and rotation of the following mother wavelet:

$\psi(\mathrm{x}, \mathrm{y})=\frac{1}{2 \pi \sigma_{\mathrm{x}} \sigma_{\mathrm{y}}} \exp \left[-\frac{1}{2}\left(\frac{\mathrm{x}^{2}}{{\sigma_{\mathrm{x}}}^{2}}+\frac{\mathrm{y}^{2}}{\sigma_{\mathrm{y}}{ }^{2}}\right)\right] \cdot \exp (\mathrm{j} 2 \pi \mathrm{Wx})$

Where $\mathrm{W}$ is called the modulation frequency. The self-similar Gabor wavelets are obtained through the generating function:

$\psi_{\mathrm{mn}}(\mathrm{x}, \mathrm{y})=\mathrm{a}^{-m} \psi(x, y)$

Where $m$ and $n$ specify the scale and orientation of the wavelet respectively, with $\mathrm{m}=0,1, \ldots \ldots \ldots . \mathrm{M}-1, \quad \mathrm{n}=$ $0,1, \ldots \ldots \ldots . . . \mathrm{N}-1$, and

$x=a^{-m}(x \cos \theta+\mathrm{y} \sin \theta)$

$y=a^{-m}(-x \sin \theta+y \cos \theta)$

Where $\mathrm{a}>1$ and $\theta=\mathrm{n} \pi / \mathrm{N}$.

The variables in the above equation are defined as follows:

$$
\begin{aligned}
& a=\left(U_{h} / U_{l}\right)^{\frac{1}{M-1}}, \\
& W_{m, n}=a^{m} U_{l}
\end{aligned}
$$

$$
\begin{aligned}
\sigma_{x, m, n} & =\frac{(a+1) \sqrt{2 \operatorname{In} 2}}{2 \pi a^{m}(a-1) U_{l}}, \\
\sigma_{y, m, n} & =\frac{1}{2 \pi \tan \left(\frac{\pi}{2 N}\right) \sqrt{\frac{U_{h}^{2}}{2 \operatorname{In} 2}-\left(\frac{1}{2 \pi \sigma_{x, m, n}}\right)^{2}}},
\end{aligned}
$$

In the implementation, we used the following constants as commonly used in the literature:

$\mathrm{U}_{1}=0.05, \mathrm{U}_{\mathrm{h}}=0.4, \mathrm{~s}$ and $\mathrm{t}$ range from 0 to 60 , i.e., filter mask size is $60 \times 60$.

\section{Texture feature Extraction}

After applying Gabor filters on the image with different orientation at different scale, we obtain an array of magnitudes:

$\mathrm{E}(\mathrm{m}, \mathrm{n})=\sum_{x} \sum_{y}\left|G_{m n}(x, y)\right|$,

$\mathrm{m}=0,1, \ldots \ldots, \mathrm{M}-1 ; \mathrm{n}=0,1, \ldots \ldots \ldots \mathrm{N}-1$.

These magnitudes represent the energy content at different scale and orientation of the image. The main purpose of texture-based retrieval is to find images or regions with similar texture. It is assumed that we are interested in images or regions that have homogenous texture, therefore the following mean $\mu_{\mathrm{mn}}$ and standard deviation $\sigma_{\mathrm{mn}}$ of the magnitude of the transformed coefficients are used to represent the homogenous texture feature of the region:

$\mu_{m n}=\frac{E(m, n)}{P X Q}$

$\sigma_{m n}=\frac{\sqrt{\sum_{x} \sum_{y}\left(\left|G_{m n}(x, y)\right|-\mu_{m n}\right)^{2}}}{P X Q}$

A feature vector $f_{g}$ (texture representation) is created using $\mu_{\mathrm{mn}}$ and $\sigma_{\mathrm{mn}}$ as the feature components [6]. For 5 scales and 4 orientations, the feature vector of length 40 is given by :

$\mathrm{f}_{\mathrm{g}}=\left\{\mu_{00}, \sigma_{00}, \mu_{01}, \sigma_{01}, \ldots \ldots \ldots \ldots, \mu_{43}, \sigma_{43}\right\}$

\subsection{Texture Similarity Measure}

The texture similarity measurement of a query image $\mathrm{Q}$ and a target image $\mathrm{T}$ in the database is defined by :

$d(Q, T)=\sum_{m} \sum_{n} d_{m n}(Q, T)$

where $d_{m n}=\frac{\left|\mu_{m n}^{Q}-\mu_{m n}^{T}\right|}{\left|\mu_{m n}^{Q}\right|+\left|\mu_{m n}^{T}\right|}+\frac{\left|\sigma_{m n}^{Q}-\sigma_{m n}^{T}\right|}{\left|\sigma_{m n}^{Q}\right|+\left|\sigma_{m n}^{T}\right|}$

If $f_{g}^{Q}=\left\{\mu_{00}, \sigma_{00}, \mu_{01}, \sigma_{01}, \ldots \ldots \ldots \ldots . . ., \mu_{43}, \sigma_{43}\right\}$ denote texture feature vector of query image and

$f_{g}^{T}=\left\{\mu_{00}, \sigma_{00}, \mu_{01}, \sigma_{01}, \ldots \ldots \ldots \ldots, \mu_{43}, \sigma_{43}\right\}$

denote texture feature vector of database image, then distance between them is given by:

$d_{2}=\sum_{i=1}^{d} \frac{\left|f_{g}^{Q}-f_{g}^{T}\right|}{\left|f_{g}^{Q}\right|+\left|f_{g}^{T}\right|}$

The Canberra distance measure is used for similarity expression.

Gabor filters are applied on the image with different scales and orientations and obtain an array of magnitudes. The mean $\mu_{\mathrm{mn}}$ and standard deviation $\sigma_{\mathrm{mn}}$ of the magnitudes are used to create a texture feature vector $f_{g}$ of length $2 * \mathrm{~m} * \mathrm{n}$. Canberra distance measure is used for computing the distance and the results of a query are displayed in decreasing similarity order.

\section{EXPERIMENTS AND RESULTS}

For evaluation of the proposed method, it has been implemented using Matlab 6.5 and Corel Image database with 1000 natural images in JPEG format of size 384 x 256 or 256 x 384 were used for testing the proposed CBIR system. In the experiment, 100 images were selected randomly; containing 
10 images in each category and the images are resized to 256 $\mathrm{x}$ 384. Within this database, it is known whether any two images are of the same category. In particular, a retrieved image is considered a match if and only if it is in the same category as the query.

The performance of a retrieval system can be measured in terms of its precision and recall. Precision measures the ability of the system to retrieve only models that are relevant, while Recall measures the ability of the system to retrieve all models that are relevant. They are defined as

Precision $=\frac{\text { Number of relevant images retrieved }}{\text { Total number of images retrieved }}=\frac{\mathrm{A}}{\mathrm{A}+\mathrm{B}}$

Recall $=\frac{\text { Number of relevant images retrieved }}{\text { Total number of relevant images }}=\frac{A}{A+C}$

Where $\mathrm{A}$ represents the number relevant images that are retrieved, B represents the number of irrelevant items and $\mathrm{C}$ the number of relevant items those were not retrieved.

The experiment was carried out with the number of retrieved images set as 10 to compute the average precision $\mathrm{P}$ of each query image.

The experiments were carried out with different values of the number of scales and orientations i.e. three scales and four orientations $(3 \times 4)[29,34]$, three scales and six orientations (3x6), four scales and five orientations $(4 \times 5)$ [30], four scales and six orientations $(4 \times 6)$ [35], five scales and four orientations $(5 \times 4)$ and five scales and six orientations $(5 \times 6)$ [33].

Among the different combinations of scales and orientations, $(3 \times 4),(4 \times 5),(4 \times 6)$ and $(5 \times 6)$ are the commonly used combinations.

Experiments were again carried out with three scales and six orientations $(3 \times 6)$ and five scales and four orientations $(5 \times 4)$. From Table 1., it is seen that the proposed five scales and four $(5 \times 4)$ gives the best performance in terms of precision than all the other combinations.

Another advantage of the proposed combination $(5 \times 4)$ is its lesser feature vector length i.e. the dimension of its texture feature vector is only 40 , in comparison with that of the $(5 \times 6)$ combination, in which the dimension of the texture feature vector is 60

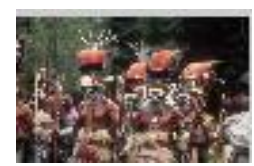

(a)

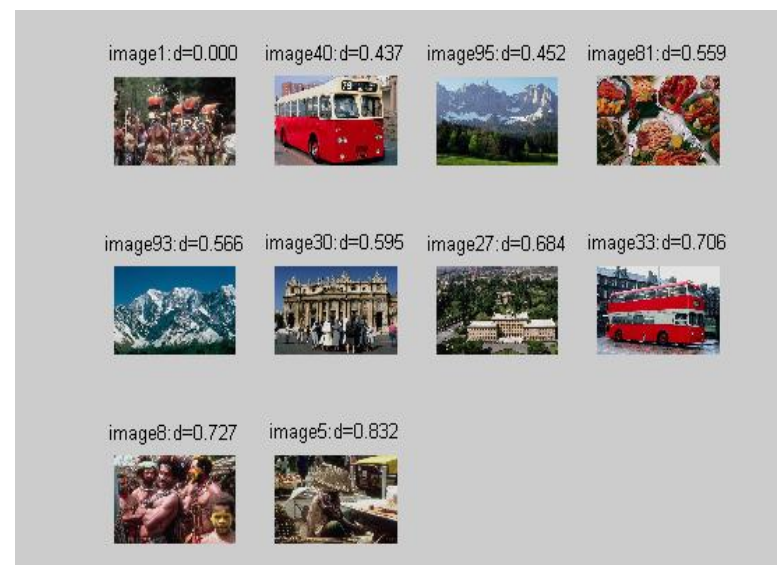

(b)

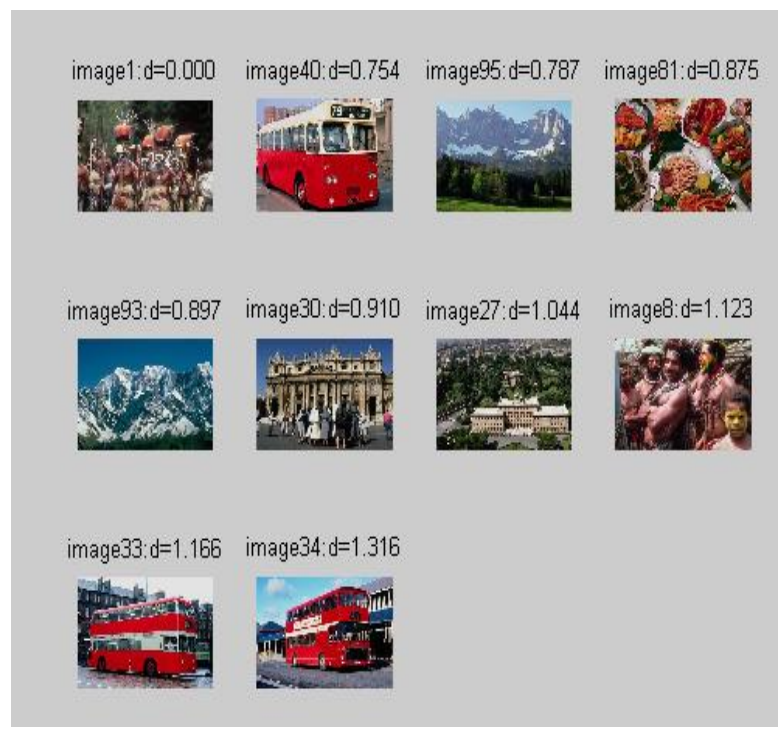

(c) 


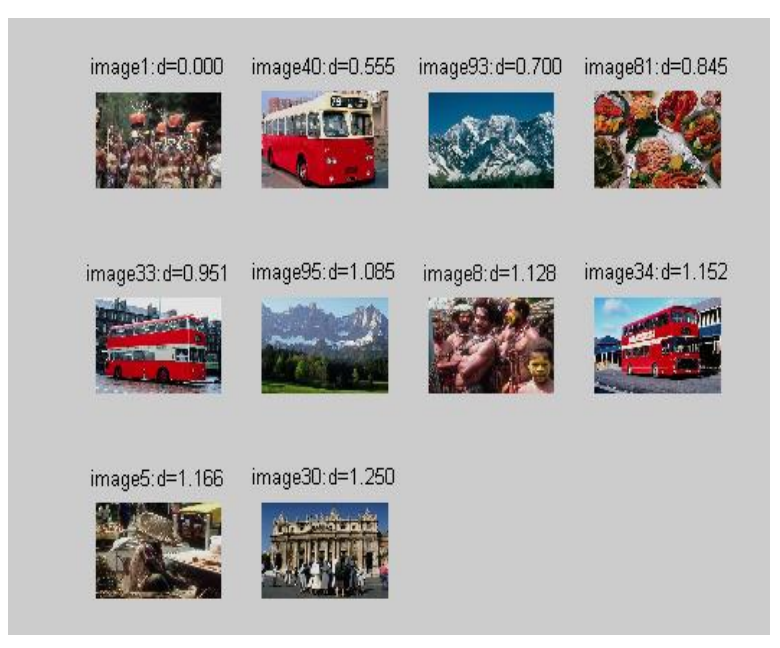

(d)

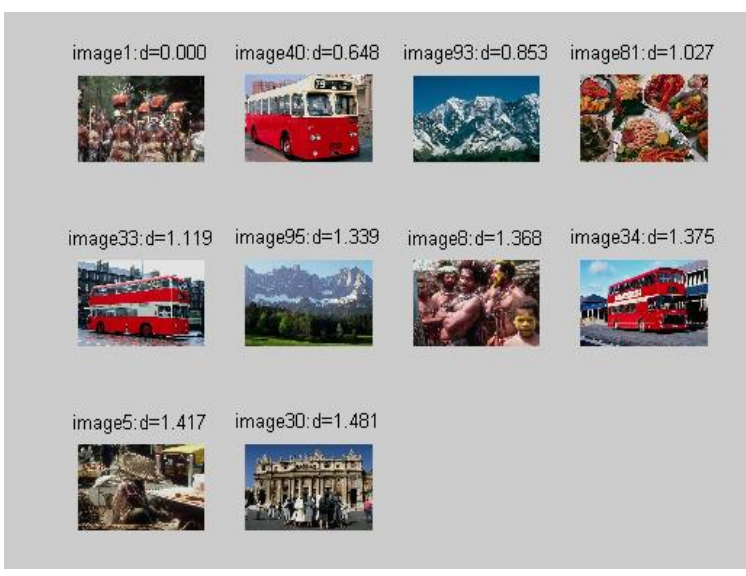

(e)

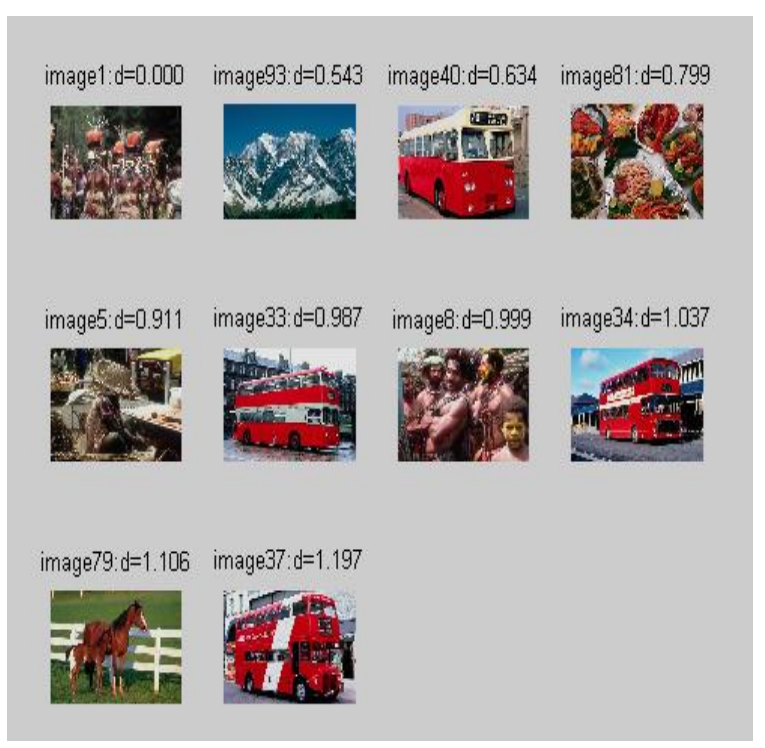

(f)

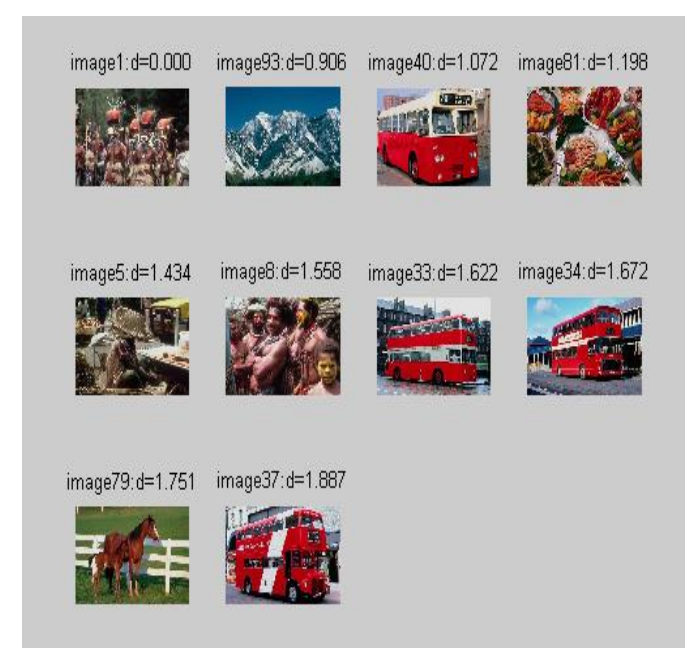

(g)

Fig. 2: For same query image (a) query image (b) result based on three scales and four orientations $(3 \times 4)$ (c) result based on three scales and six orientations (3x6) (d) )result based on four scales and five orientations $(4 \times 5)$ (e) result based on four scales and six orientations $(4 \times 6)$ (f) result based on five scales and four orientations $(5 \times 4)(\mathrm{g})$ result based on five scales and six orientations $(5 \times 6)$.

Table 1. Precision of retrieval for top 10 images using Gabor filter. 3 scales and 4 orientations (3x4); 3 scales and 6 orientations $(3 \times 6) ; 4$ scales and 5 orientations $(4 \times 5) ; 4$ scales and 6 orientations (4x6); 5 scales and 4 orientations $(5 \times 4) ; 5$ scales and 6 orientations $(5 \times 6)$.

\begin{tabular}{|c|c|c|c|c|c|c|}
\hline \multirow{2}{*}{$\begin{array}{l}\text { Image } \\
\text { class }\end{array}$} & & \multicolumn{5}{|c|}{ Average Precision } \\
\hline & $3 \times 4$ & $3 \times 6$ & $4 \times 5$ & $4 \times 6$ & $5 \times 4$ & $5 \times 6$ \\
\hline Africa & 0.31 & 0.28 & 0.35 & 0.35 & 0.36 & 0.37 \\
\hline Beach & 0.25 & 0.26 & 0.26 & 0.27 & 0.30 & 0.28 \\
\hline Building & 0.31 & 0.30 & 0.31 & 0.32 & 0.29 & 0.29 \\
\hline Buses & 0.36 & 0.37 & 0.32 & 0.33 & 0.31 & 0.30 \\
\hline Dinosaur & 0.99 & 0.99 & 0.99 & 0.99 & 0.99 & 0.99 \\
\hline Elephant & 0.39 & 0.38 & 0.37 & 0.37 & 0.36 & 0.36 \\
\hline Flowers & 0.79 & 0.71 & 0.75 & 0.75 & 0.76 & 0.76 \\
\hline Horses & 0.27 & 0.27 & 0.27 & 0.26 & 0.29 & 0.29 \\
\hline Food & 0.40 & 0.41 & 0.41 & 0.41 & 0.40 & 0.40 \\
\hline Mountain & 0.20 & 0.21 & 0.20 & 0.19 & 0.23 & 0.24 \\
\hline $\begin{array}{l}\text { Average } \\
\text { Precision } \\
(\%)\end{array}$ & 42.7 & 41.8 & 42.3 & 42.4 & 42.9 & 42.8 \\
\hline
\end{tabular}




\section{CONCLUSION}

In this study, experiments were carried out to extract texture features from natural images using Gabor filter at different values of the number of scales(S) and the number of orientations $(\mathrm{K})$. The commonly used scales and orientations as published by research scholars are 3 scales and 4 orientations, 4 scales and 5 orientations, 4 scales and 6 orientations and 5 scales and 6 orientations. The experiments show that for natural images, the proposed 5 scales of frequency and four orientations gives better result in terms of precision than the other different values of scales and orientations.

\section{REFERENCES}

[1] Brodatz, P. 1966. "Textures: A Photographic Album for Artists and Designers", Dover Publication, New York.

[2] Eakins J.P. and Graham E.M. “ Content-based Image Retrieval: A Report to the JISC Technology Applications Program".http://www.unn.ac.uk/iidr/research/cbir/report. html.

[3] Tamura, H., Mori, S., Yamawaki, T. 1976. "Texture features corresponding to visual perception", IEEE Trans. On Systems, Man and Cybernetics. 6(4): 460-473.

[4] Niblack, W., Barber, R., Equitz, W., Flickner, M., Glasman, E., Petkovic, D., Yanker, P., Faloutsos, C. and Taubin, G. 1993. "The QBIC Project: Querying Images by Content Using Color, Texture, and Shape". Proc. Of the Conference Storage and Retrieval for Image and Video Databases, SPIE vol. 1908, pp. 173-187.

[5] Liu, F. and Picard, R.W. 1996. "Periodicity, directionality and randomness: Wold features for image modelling and retrieval", IEEE Transactions on Pattern Analysis and Machine Intelligence 18(7): 722-733.

[6] Manjunath, B.S. and Ma, W.Y. 1996. "Texture Features for browsing and retrieval of image data", IEEE Transactions on Pattern Analysis and Machine Intelligence, Vol.18, No.8, pp. 837-842.

[7] Kaplan, L.M., Murenzi, R., Namuduri, K. R. 1998. "Fast texture database retrieval using extended fractal features," in Storage and Retrieval for Image and Video Databases, San Jose, CA, vol. 3312, pp. 162-175.

[8] Smith, J.R. 1997. "Integrated Spatial and Feature Image System: Retrieval, Analysis and Compression", Ph.D. thesis, Columbia University.

[9] Deng, Y. 1999. "A Region Representation for Image and Video Retrieval", Ph.D. thesis, University of California, Santa Barbara.

[10] Ma, W.Y. 1997. "Netra: A Toolbox for Navigation Large Image Databases", Ph.D. thesis, University of California, Santa Barbara.

[11] Jeanin $\quad$ S. $\quad$ (ed.), $2000 \quad$ "ISO/IEC JTCI/SC29/WG11/N3321: MPEG-7 Visual Part eXperimentation Model Version 5.0", Nordwijkerhout.

[12] Dimai, A. 1999. "Rotation Invariant Texture Description using General Moment Invariants and Gabor Filters", In Proc. Of the 11th Scandinavian Conf. on Image Analysis. Vol I, pp. 391-398.

[13] Daugman J.G. 1985. "Uncertainty relation for resolution in space, spatial frequency, and orientation optimized by two-dimensional visual cortical filters", Journal of The Optical Society of America:2(7):1160-1169.

[14] Moores, C. 1951 "Datacoding Applied to Mechanical Organization of Knowledge", American Documentation, Vol. 2, pp. 20-32.

[15] Datta, R., Joshi, D., Li, J., Wang, J.Z. 2008. "Image retrieval: ideas, influences, and trends of the new age ", ACM Computing Surveys 40(2), pp 1-60.

[16] Gudivada, V.N. and Raghavan, V.V. 1995. "Content based image retrieval systems", IEEE Computer, Vol 28, No.9, pp.18-22.

[17] Rui, Y., Huang, T.S., Ortega, M. and Mehrotra, S. 1998. " Relevance feedback : a power tool for interactive content based image retrieval ",IEEE Circuits and Systems for Video Technology, Vol. 8, No. 5, pp. 644655 .

[18] Swets, D. and Weng, J. 1999. "Hierarchical discriminant analysis for image retrieval", IEEE "PAMI, Vol. 21, No. 5, pp. 386-400.

[19] Zhang, H. and Zhong, D. 1995. "A scheme for visual feature based image retrieval", Proc. SPIE storage and retrieval for image and video databases.

[20] Yu-guang, Ye. 2007. "Research of image Retrieval based on fusing with multi-character", Hua Qiao University, pp. $14-16$

[21] Singha, M, Hemachandran, K. and Paul, A. 2012 “ Content - based image retrieval using the combination of fast wavelet transform and the color histogram", IET Image Process., pp. 1-6

[22] Smeulders, A.M., Worring, M., Santini, S., Gupta, A. and Jain, R. . 2000. "Content-based image retrieval at the end of the early years", IEEE Trans Pattern Anal Machine Intell 22: pp.1349-1380.

[23] Choras, R. 2003. "Content-based image retrieval using color, texture, and shape information", In. Sanfeliu, Riuz-Shulcloper J. (eds) Progress in pattern recognition, speech and image analysis. Springer, Heidelberg.

[24] Corners, R. and Harlow, C. 1980. "A theoretical comparison of texture algorithms", IEEE Trans Pattern Anal Machine Intell 2: pp.204-222.

[25] Howarth, P. and Ruger, S. "Evaluation of texture features for content based image retrieval", In: Enser P. et al. (eds) Image and video retrieval. Springer LNCS 3115:pp.326-334.

[26] Choras, R.S., Andrysiak, T. and Choras, M. 2007. "Integrated color, texture and shape information for content-based image retrieval", Pattern Anal Applic. 10: 333-343.

[27] Huang, Z.C., Chan, P.P.K., Ng, W.W.Y., Yeung, D.S 2010. "Content-based image retrieval using color moment and Gabor texture feature", in Poceedings of the IEEE Ninth International Conference on Machine Learning and Cybernetics, Qingdao, pp. 719-724.

[28] Maheshwari, M. , Silakari, S. and Motwani, M. 2009. "Image Clustering using Color and Texture", Computational Intelligence, Communication Systems and Networks, pp. 403-408. 
[29] Gali, N., Venkateshwar Rao, B., Subhani Shaik, A. 2012 "Color and Texture Features features for Image Indexing and Retrieval", International Journal of Electronics Communication and Computer Engineering Volume 3, Issue (1) NCRTCST, ISSN 2249-071X.

[30] Ashok Kumar, D., Esther, J. 2011 “ Comparative Study on CBIR based by Color Histogram, Gabor and Wavelet Transform “, IJCA,Vol 17, No.3, pp. 17-44.

[31] J. Zhang, G. Li, S. He, "Texture-Based Image Retrieval by Edge Detection Matching GLCM", The 10th IEEE International Conference on High Performance Computing and Communications.

[32] Clause, D.A., Jerni, M. Ed., Gan, 2000. "Designing Gabor filters for optional texture separability", Pattern Rcognition, 33, pp. 1835-1849.
[33] Zhang, D., Wong, A., Indrawan, M., Lu, G. 2000. "Content- based Image Retrieval Using Gabor Texture Features", IEEE Pacific - Rim Conference on Multimedia, University of Sydney, Australia.

[34] Murala, S., Gonde, A.B., Maheshwari, R.P. 2009 . "Color and Texture Features for Image Indexing and Retrieval", IEEE International Advance Computing Conference, Patiala, India, 6-7 March.

[35] Michael Eziashi Osadebey, 2006. “ Integrated ContentBased Image Retrieval using Texture, Shape and Spatial Information", Master Thesis Report in Media Signal Processing, Department of Applied Physics and Electronics, Umea University, Umea, Sweden. 